\title{
Atomic scale step structure and orientation of a curved surface $\mathrm{ZnO}$ single crystal
}

Elin Grånäs, ${ }^{1}{ }^{1}$ a) Björn Arndt, ${ }^{1}$ Christoph Seitz, ${ }^{1}$ Michael Wagstaffe, ${ }^{1}$ and Andreas

Stierle $\mathrm{e}^{1,2, \mathrm{~b})}$

1) Deutsches Elektronen-Synchrotron (DESY), 22607 Hamburg,

Germany

2) Fachbereich Physik, Universität Hamburg, 20355 Hamburg,

Germany

(Dated: 21 January 2020)

We have investigated the surface structure of a curved $\mathrm{ZnO}$-crystal, going from the (0001)-facet at $0^{\circ}$ miscut to the (1014)-facet at a miscut of $24.8^{\circ}$ using scanning tunneling microscopy and low energy electron diffraction. We find that the surface separates locally into (0001)-terraces and (1014)-facets, where the ratio between the facets depends on the miscut angle. In X-ray photoemission spectroscoy (XPS) the intensity of an O 1s component scaling with the step density of the surface is observed. No other facets were observed and the surface maintains a high degree of order over all angles. Such a curved $\mathrm{ZnO}$ crystal can be used for systematic studies relating the step density to the chemical reactivity using XPS to probe the curved surface at different positions.

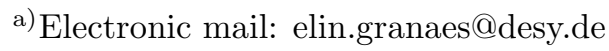

b)Electronic mail: andreas.stierle@desy.de
} 


\section{INTRODUCTION}

The low-coordinated atoms on step sites often play an important role in the catalytic, electrical- and optical- properties of surfaces ${ }^{1,2}$. Vicinal surfaces, providing well-ordered step arrays, are often used as model systems to systematically study the influence of such sites on the surface chemistry ${ }^{3-8}$. Further, vicinal surfaces are an interesting playground as templates for growing nanostructures ${ }^{9-12}$.

Vicinal surfaces are defined by the miscut with respect to a high symmetry direction, where generally a higher miscut angle results in a denser step array. One way of exploring properties of vicinal surfaces that depend on step density and orientation is to use curved crystals, for which a smooth variation of the miscut angle is provided. In this way 'families' of vicinal planes can be studied, providing a coherent picture of the physical- and chemical properties that depend on the step density or orientation. The use of curved crystals, as opposed to several vicinal crystals, circumvents problems with heterogeneity between samples through different contaminants or reduction states in oxide crystals. The approach of using curved crystals has proven useful for metals in numerous studies ${ }^{1,13-18}$. Curved oxides on the other hand, despite also being important in many applications ${ }^{19}$, are very sparsely studied. A study of curved rutile $\mathrm{TiO}_{2}{ }^{20}$, however, has demonstrated that this concept can be similarly applied to oxide surfaces. For oxides such as $\mathrm{ZnO}$ many of the surface orientations will be unstable due to the non-zero dipole moment and the corresponding surface polarity ${ }^{21}$. This will result in deviations from the smooth variation in step density observed for most metals, as faceting and reconstructions are expected to occur in order for the surface to be charge neutral.

In this work we studied a $\mathrm{ZnO}$ single crystal curved from (0001) to (1014); recently the vicinal $\mathrm{ZnO}(10 \overline{1} 4)$ surface was identified as a stable vicinal orientation of $\mathrm{ZnO} .{ }^{22-24}$ This surface has a charge neutral unit cell and a high step-density, providing an abundance of highly reactive low-coordinated sites. Using scanning tunneling microscopy (STM), low energy electron diffraction (LEED) and x-ray photoelectron spectroscopy (XPS) we strive to obtain a more thorough understanding of the faceting and reconstructions in this family of vicinal planes, with the future aim to use curved $\mathrm{ZnO}$ crystals to study step dynamics and the influence of steps on the catalytic properties of $\mathrm{ZnO}$. 


\section{EXPERIMENTAL DETAILS}

The curved crystal was cleaned through cycles of sputtering $\left(\mathrm{Ar}^{+}, 500 \mathrm{eV}\right)$ at room temperature and subsequent annealing at $650{ }^{\circ} \mathrm{C}$, according to a recipe developed for the single crystal (1014) surface ${ }^{24}$. The sputtering was performed at grazing angle and along the step direction of the crystal. The clean crystal contained no impurities as seen by XPS measurements.

The STM measurements were performed in the VT AFM/STM at the DESY NanoLab ${ }^{25}$. All measurements were performed at room temperature in constant current mode using a etched W-tips (Ar-sputter and annealed). The images were post processed using Gwyddion $^{26}$. The image slope at the (0001)-oriented side of the crystal [see Figure 1(a)] was determined to be $0.8 \pm 0.5^{\circ}$. For images measured elsewhere on the crystal the average image slope was used to determine the local miscut angle, i.e. the position on the crystal. This was further correlated with the measurement position as determined by an optical microscope mounted on the chamber. LEED measurements were performed in a different part of the UHV-system that was accessed through in-vacuum transfer. The LEED electron beam had a diameter of $0.3 \mathrm{~mm}$. Both UHV-chambers have a base pressure better than $5 \cdot 10^{-11}$ mbar.

The XPS measurements were carried out at the PEARL beamline at the Swiss Light Source $^{27}$. The $\mathrm{O} 1 \mathrm{~s}$ spectra were measured at a photon energy of $650 \mathrm{eV}$ in five different spots evenly spread over the sample surface. The measurements were performed at $40^{\circ}$ grazing incidence, the curvature of the sample was taken into account so that the incidence angle was the same in all spots, ensuring a constant probing depth. The beamsize was approximately $190 \mu \mathrm{m} \times 70 \mu \mathrm{m}$.

\section{RESULTS AND DISCUSSION}

Figure 1(a) shows a side view of the curved $\mathrm{ZnO}$ crystal used in this study. The sample is a cylindrical section cut, with the (0001) plane on one side, going to a total miscut angle of $\alpha=24.8^{\circ}$, corresponding to the (1014) surface. The $(\overline{1} 2 \overline{1} 0)$ direction runs perpendicular to the curvature. The angle of the curvature is such that we expect (0001)-oriented terraces with decreasing width towards the (1014) side of the crystal, running through a whole series 
(a) (0001)

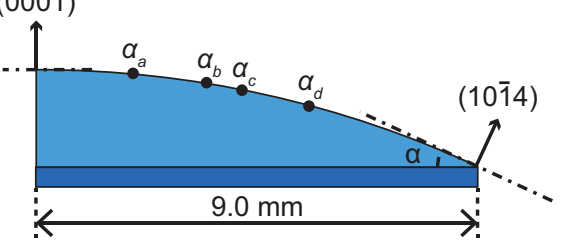

$\begin{array}{lll}\text { (b) } \mathrm{ZnO}(0001) \quad \odot \mathrm{Zn} & \text { (c) } \mathrm{ZnO}(10 \overline{1} 4)\end{array}$
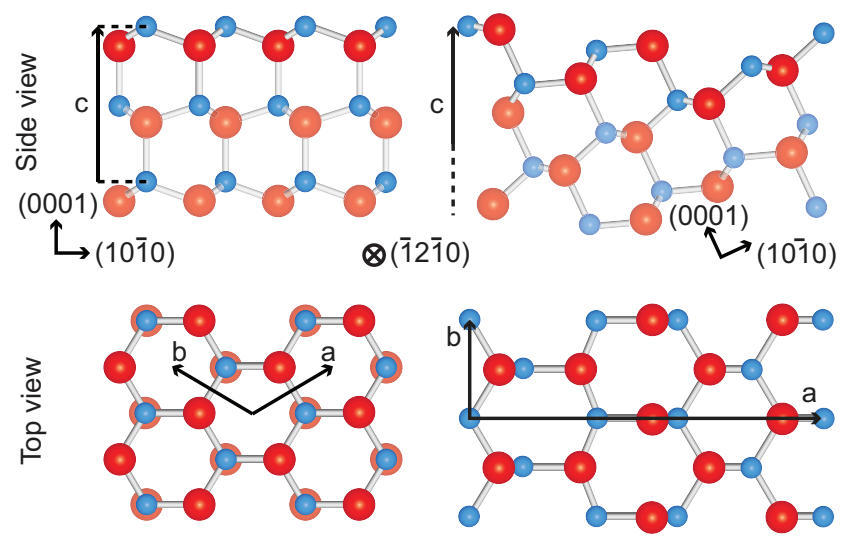

FIG. 1. (a) Sketch of the curved $\mathrm{ZnO}$ crystal. $\alpha_{a}-\alpha_{d}$ indicate the position of the STM images shown in Figure 3. (b) and (c): Top and side views of atomic models for the $\mathrm{ZnO}(0001)-$ and (1014)-surfaces. $a, b$, and $c$ indicate the unit cell for the two surfaces. For the (0001)-surface $a=b=3.25 \AA$ and $c=5.205 \AA$ and for the (1014)-surface $a=12.4 \AA, b=3.25 \AA$ and $c=40.32$ $\AA$.

of vicinal planes. An atomic model of the (0001)-surface is shown in Figure 1(b). The atomic scale structure of the vicinal (1014)-surface we study in a forthcoming publication ${ }^{24}$. Using a combination of experimental and theoretical methods we find that $\mathrm{ZnO}(10 \overline{1} 4)$ is a stable long-range ordered, non-polar facet with a high step-density and a uniform bulk terminated surface as depicted in Figure 1(c).

LEED images from the (0001)- $\left[\alpha_{0}=0^{\circ}\right]$ and the (1014)-side $\left[\alpha_{1}=24.8^{\circ}\right]$ of the crystal are shown in Figure 2(a) and (b), respectively. The LEED image at $\alpha_{0}$ shows a hexagonal pattern as expected for a (0001) surface while at $\alpha_{1}$ all the fundamental signals exhibit splitting typical for periodic arrays of steps on the surface ${ }^{28}$. The separation between the satellite signals are the reciprocal of the average periodicity of the vicinal surface: $2 \pi / D$, where $D$ is the real space periodicity. The LEED pattern at $\alpha_{1}$ is in good agreement with what we have observed for $\mathrm{ZnO}(10 \overline{1} 4)$ single crystals, to be presented in a forthcoming publication $^{24}$. 

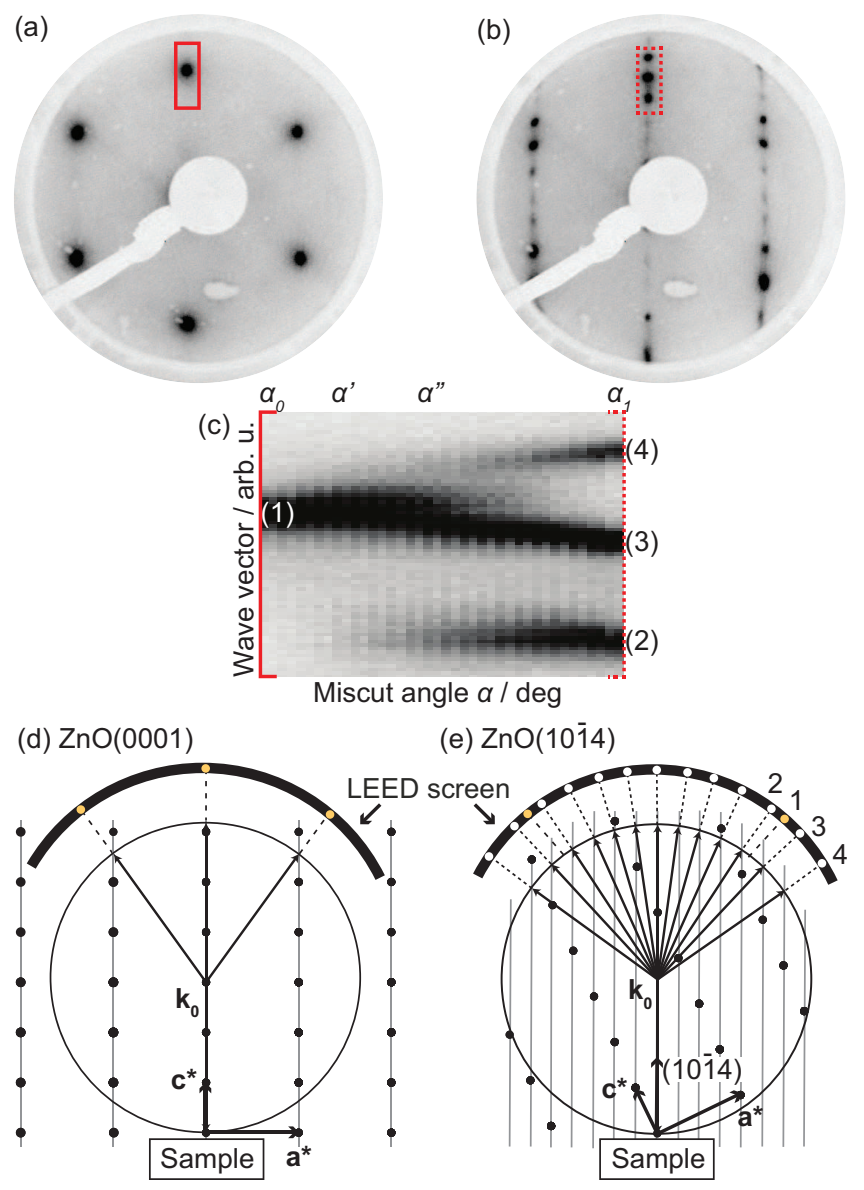

FIG. 2. (a) LEED pattern from the (0001)-side of the crystal, $\alpha_{0}$. (b) LEED pattern from the (1014)-side of the crystal, $\alpha_{1}$. (c) Cut out from LEED images taken across the surface as indicated by the red rectangles in (b) and (c). All LEED images were taken at $53 \mathrm{eV}$. (d) LEED diffraction geometry and reciprocal lattice for the (0001) oriented area of the curved $\mathrm{ZnO}$ crystal in the $(0001) /(10 \overline{1} 0)$ plane. $a^{*}=2 \pi / \mathrm{a}$ and $\mathrm{c}^{*}=2 \pi / \mathrm{c}$, a and $\mathrm{c}$ are the bulk lattice constants of $\mathrm{ZnO}$. The direction of the outgoing electron beams (dashed lines) are constructed by the intersection of the Ewald sphere with the reciprocal lattice rods running perpendicular to the surface. Open circles on the LEED screen indicate positions of reflections. Filled circles indicate reciprocal lattice points of bulk $\mathrm{ZnO}$. (e) LEED diffraction geometry of the (1014) oriented area of the curved $\mathrm{ZnO}$ crystal in the $(0001) /(10 \overline{1} 0)$ plane. Reflections (1)-(4) on the LEED screen correspond to the signals (1)-(4) in Figure 2(d). The dashed line and colored spot indicates the direction of outgoing beams from larger (0001) oriented facets. 
The relatively large sample size and radius of curvature enables scanning of the sample through the electron beam and thus probing the step periodicity across the surface. In Figure 2(c) cuts through the area marked in panels (a) and (b) are displayed for LEED images taken across the surface. The crystal position was adjusted by $0.5 \mathrm{~mm}$ in the direction of the curvature between the images. Initially, i.e. at $\alpha_{0}$, a single signal, (1), from the (0001) surface is seen. When going to higher miscut angles the FWHM of the signal is increasing while the position remains unchanged. At $\alpha^{\prime}=5^{\circ}$ a satellite signal, (2), appears and increases in intensity as the miscut angle increases. Meanwhile the first signal splits up in two, one corresponding to the (0001) signal which fades out at $\alpha^{\prime \prime} \approx 12^{\circ}$ and one signal, (3), that shifts in position as the miscut angle increases. At approximately the same angle another satellite signal, (4), also appears. These changes in the LEED signals from the surface can be understood by inspecting the diffraction geometry and reciprocal lattice for the (0001) and (1014) oriented areas, as shown in Figure 2(d) and (e). The signal (1), seen in both panel (d) and (e), corresponds to (0001) oriented areas on the surface, which have a lateral size which is larger that the transversal electron coherence length. This signal does not shift in the LEED pattern in Figure 2(c) and it fades out at a position on the crystal where no larger (0001) areas are observed by STM (see Figures 3 and 5 and discussion thereof). The distance between spots (2), (3) and (4) is continuously changing as a function of the decreasing average size and distance of (0001) and (1014) surface areas. We do not assign this behavior to a continuous change of the average step width but rather to an interference effect occuring in systems of statistical occurrence of two different periodicities (see discussion below $)^{29}$.

Through STM measurements we have further characterized the evolution of the step structure along the curvature of the sample. In Figure 3(a)-(d) four representative STM images are shown, taken at the positions indicated in Figure 1(a). At low miscut angles, as exemplified by image (a), the surface displays wide, kinked, (0001)-terraces which are occasionally separated by double steps. Moving to higher miscut angles, as in image (b) at $\alpha=9^{\circ}$ and (c) at $\alpha=11^{\circ}$, the (0001)-terraces become more and more narrow and straight while step bunches appear, all aligned perpendicular to the (1010)-direction. Continuing along the curvature of the sample the terrace width, $d_{\text {terr }}$, steadily decreases while the extent of the step bunches increases. Already around $\alpha=15^{\circ}$ the surface is consisting of arrays of steps occasionally interrupted by somewhat wider terraces rather than terraces 

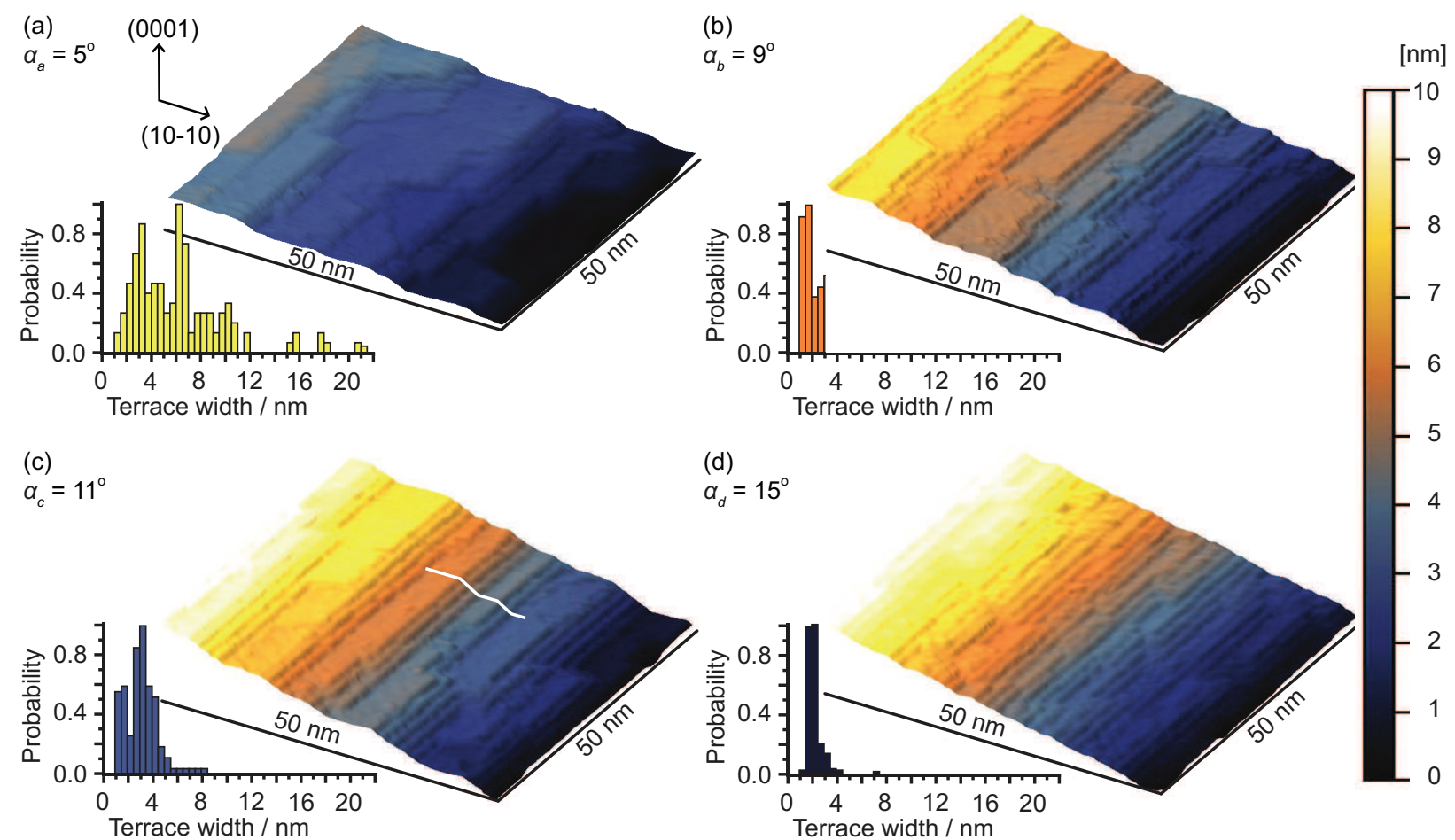

FIG. 3. (a)-(d): STM images taken at four different angles $\alpha$, as indicated Figure 1(a). All images are $50 \times 50 \mathrm{~nm}^{2}$. The histograms show the distribution of widths for several $200 \times 200 \mathrm{~nm}^{2}$ images taken at $\alpha \pm 1^{\circ}$. STM measurement conditions for all images: $\mathrm{U}=2.5 \mathrm{~V}, \mathrm{I}=0.05 \mathrm{nA}$. The white line in panel (c) indicate the line scan in Figure 4(a).

separated by step bunches, as seen in image (d).

These step bunches always have a step periodicity of $d_{\text {step }}=14 \pm 1 \AA$ and have on average a step height, $h$, of $4.9 \pm 0.03 \AA$. Both the step periodicity and height are close to what is expected for a $\mathrm{ZnO}(10 \overline{1} 4)$-surface: $d_{\text {step }}=12.4 \AA$ and $h=c=5.2 \AA(a=3.25 \AA)$. In Figure 4(a) a line scan from the STM image shown in Figure 3(c) is shown. Here $d_{\text {step }}$ and $h$ are indicated as well as the (0001)-terrace width $d_{\text {terr }}$. The atomic model of the surface is shown under the line scan, confirming that the observed steps match a (1014) structure. In panel (b) the average (0001)-terrace width, $\left\langle d_{t e r r}\right\rangle$, is displayed as a function of the miscut angle. The analysis for Figure 4(b) and 5 was carried out on images of $200 \times 200 \mathrm{~nm}^{2}$, except for at the highest angles where the analyzed area was reduced to approximately half. Measurements from different occasions and after different number of preparation cycles are fully consistent with eachother. In the range $\alpha=5-15^{\circ}$ the decrease in (0001)-terrace width is almost linear, reflecting the statistical occurrence of (0001) and (1014) oriented 

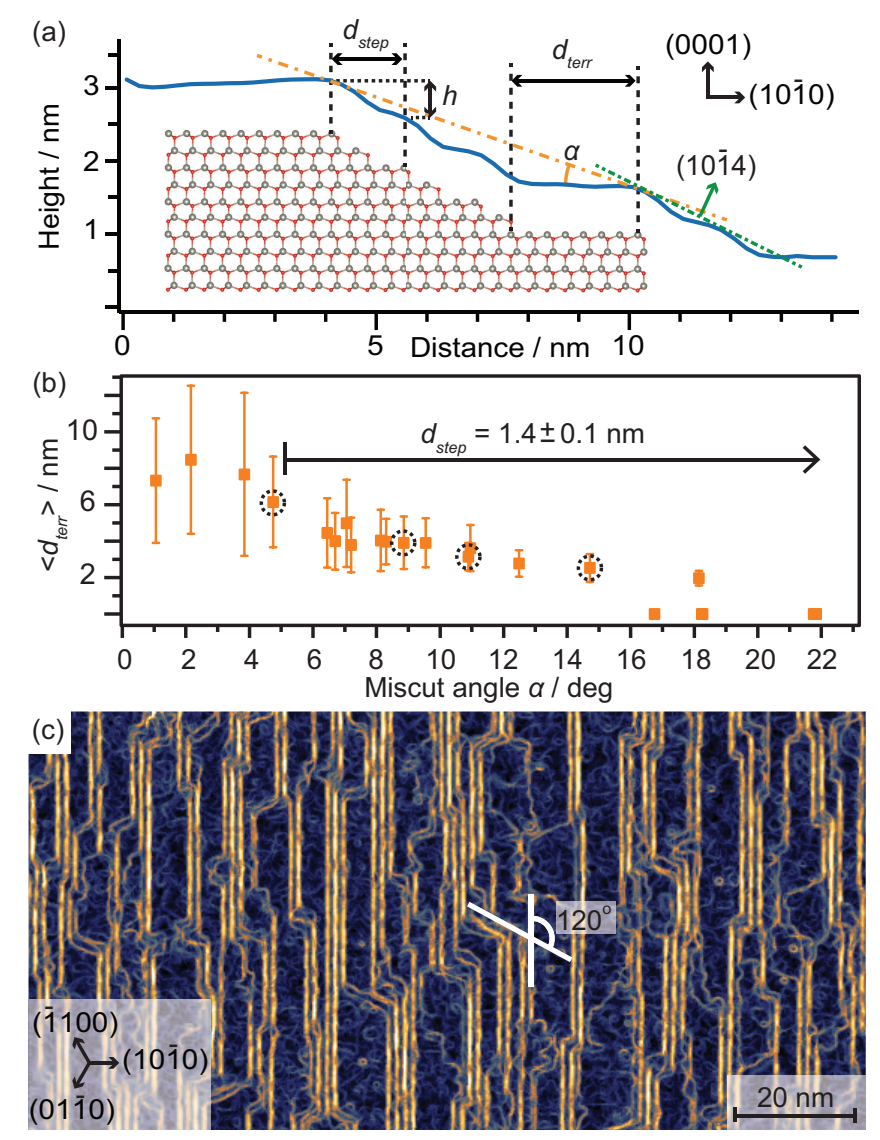

FIG. 4. (a) Line scan along the white line indicated in Figure 3(c) with the corresponding atomic model. (b) The average width of the (0001)-terraces, $\left\langle d_{t e r r}\right\rangle$, as a function of the miscut angle with the standard deviation indicated. The encircled points corresponds to the images in Figure 3. (c) STM image filtered to enhance height changes. $\alpha=9^{\circ}$. Conditions for STM imaging: $U=2.5 \mathrm{~V}$, $I=0.05 \mathrm{nA}$.

surface areas.

In Figure 4(c) an STM image taken at $\alpha=9^{\circ}$ is shown. The image was filtered to enhance height changes, height changes are displayed as bright while flat areas are dark. First focusing on the step bunches, the edges of these are straight and aligned with the ( $\overline{1} 2 \overline{1} 0)$ direction perpendicular to the step direction. The morphology of the wider terraces however differs from that of the step bunches, displaying kinked edges. Analyzing the direction of these short edges they are seen to be rotated $120^{\circ}$ from the long edge, aligning with the $(1 \overline{1} 00)$ and $(01 \overline{1} 0)$ directions as would be expected from a hexagonal surface such as $\mathrm{ZnO}(0001)$. The step height in the $(1 \overline{1} 00)$ and $(01 \overline{1} 0)$ directions often correspond to $c / 2$, rather than $c$ as is observed for the (1014) step bunches. This single layer step height in the short directions 
of the terraces is consistent with the height observed for $\mathrm{ZnO}(0001)$ surfaces, in contrast to $\mathrm{ZnO}(000 \overline{1})$ surfaces that typically have a step height corresponding to $c^{30}$.
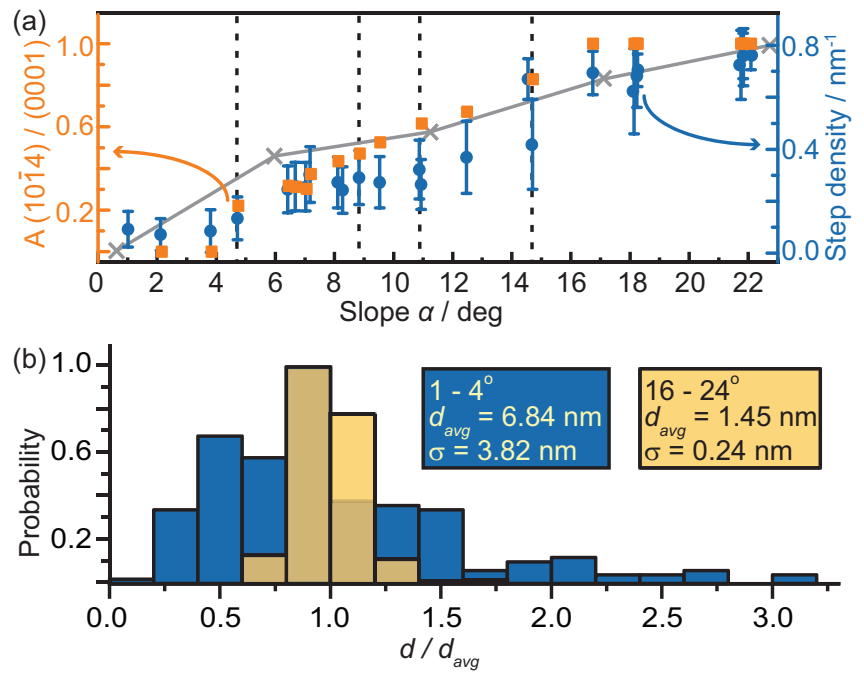

FIG. 5. (a) Area fraction, A, of the (1014)-facet relative to the (0001)-facet [left y axis] and the density of steps [right y axis] as a function of the slope angle $\alpha$. The dashed lines indicate the points corresponding to the images in Figure 3. The grey crosses correspond to the area under the XP-difference spectra displayed in Figure 6(b) in arbitrary units (normalised to the highest value).

(b) Reduced terrace width histograms for low and high miscut angles.

Further proof for that the step bunches correspond to (1014)-facets comes from the angle of the facets relative to the (0001)-terraces. The angle is measured to be $20 \pm 2^{\circ}$, which is close to the theoretical angle of $24.8^{\circ}$ for $\mathrm{ZnO}(10 \overline{1} 4)$ and to what has been observed for (1014)-facets on $\mathrm{ZnO}(0001)$ surfaces previously ${ }^{22}$. Through analyzing the facet angles the area fraction of the (1014)-facets versus the (0001)-facets as a function of the miscut angle can be determined, as displayed in Figure 5(a). We see that at $\alpha=5^{\circ}$, the STM

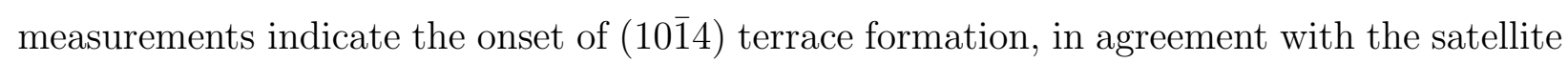
spot appearing in the LEED singal at $\alpha^{\prime}$ [see Figure 2(c)]. At $\alpha^{\prime \prime}=12^{\circ}(0001)$-signal has faded in the LEED measurements, while in the STM measurements we see that the (1014)facet is dominating in this range and the average width of the (0001)-facets is only $2.8 \pm 0.7$ nm. Also displayed in Figure 5(a) is the average step density calculated from the measured $d_{\text {step }}$ and $d_{\text {terr }}$. This is in good agreement with the area fraction estimated from the facet angle, which similarly increases linearly between a miscut angle of 5 and $15^{\circ}$.

The (0001)- and (1014)-facets together make up $62 \pm 5 \%$ of the surface area in this range, 
with the remaining $38 \%$ being made up of side facets from the kinked terraces at the lower miscut angles as well as some less ordered areas. At high miscut angles imaging was more challenging and the analyzed areas are much smaller, leading to poor statistics concerning how much of the surfac area that is made up by (0001)- and (1014)-facets. The driving factor to form the charge neutral (1014) nano-facets already at such low miscut angles as $5^{\circ}$ is the non-zero dipole moment of the (0001)-facets. Similarly, faceting into (1014) was observed for annealed $\mathrm{ZnO}(0001)$-surfaces ${ }^{22}$. Such faceting is expected for surfaces where other forces than step-interactions contribute to the energy balance, and has been observed for example for curved $\mathrm{Au}(111)^{18,31}, \mathrm{Ni}(111)^{14}, \mathrm{GaAs}(001)^{32}$ and $\mathrm{TiO}_{2}(110)^{20}$. The formation of facets also often depends on the direction of the miscut ${ }^{14,15,18}$.

The distribution of terrace widths around the mean value, $d / d_{\text {avg }}$, is shown in Figure $5(\mathrm{~b})$ for the lowest $\left(1-4^{\circ}\right)$ and highest $\left(16-23^{\circ}\right)$ miscut angles. For the lowest angles the fluctuation of terrace width is large and with an asymmetric broadening towards larger terraces. In contrast, at the higher angles the distribution is very narrow and peaked around the mean value which corresponds to the periodicity of the (1014)-surface. These distributions can be correlated with the LEED measurements, where the sharpness of the signal reflects the degree of order on the surface. In Figure 2(c) the FWHM of the LEED signal (1) is significantly broader than any of the signals are at $\alpha_{1}$, in agreement with the broad terrace distribution seen in the STM images. The absence of satellite spots in the LEED is in line with expectations for such a wide distribution of terrace widths ${ }^{33}$. For the well defined vicinal surface we observe at high miscut angles satellite spots as well as a narrower FWHM of the signals are expected in the LEED, in line with what we observe. Both the wide distribution of terrace width and the kinked edges of the terraces indicate a low interaction between the steps at low miscut angles. At these low step densities the shape is instead governed by the energy of the step edge and other favorable edge orientations may appear ${ }^{34}$. Meanwhile the straight edges and well defined terrace width of the (1014)-facets indicates a strong step-step interaction and an electrostatic driven stabilization of this zero-dipole moment surface orientation.

We also characterised the curved crystal with XPS. Measurements were performed in five points on the surface, as indicated in the inset in Figure 6(a). The O 1s spectra displayed in Figure 6(a) were taken at p1, corresponding to the (0001)-side of the crystal, and at p5, corresponding to the (1014)-side. The spectra were normalised to the peak and a linear 

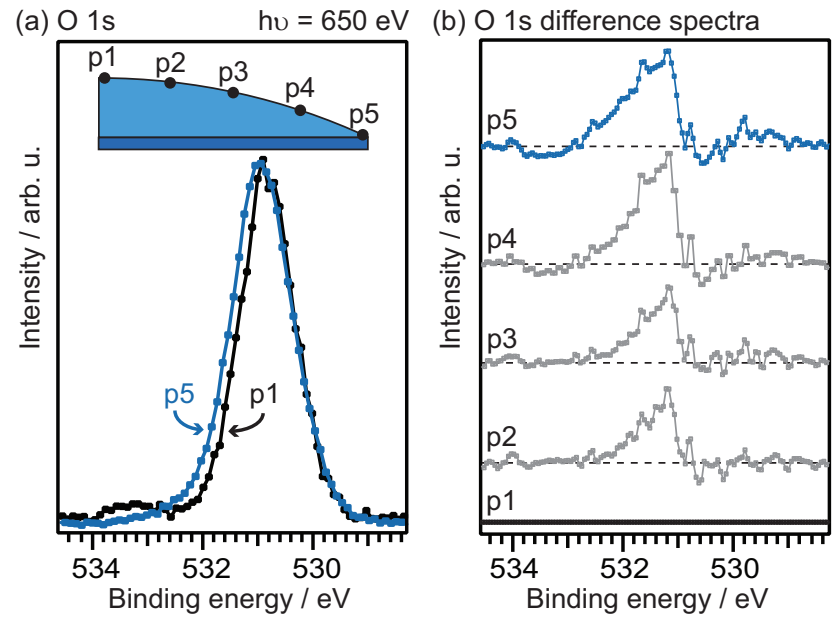

FIG. 6. (a) O 1s spectra taken at the the points indicated in the sketch. p1 (black) corresponds to the (0001)-side and p5 (blue) to the (1014)-side of the crystal. (b) Differences of the O 1s spectra taken at the respective point, calculated as $i$-p1.

background was subtracted. In $\mathrm{p} 1$ the $\mathrm{O}$ 1s spectrum shows one symmetric peak centered at $530.95 \mathrm{eV}$. In comparison the spectrum taken in p5 is significantly wider on the high binding energy side. This widening we also observe for the single crystal (1014)-surface, where we assign it to under coordinated O-atoms ${ }^{24}$. The small peak around $533.4 \mathrm{eV}$ observed in $\mathrm{p} 1$ is due to small amounts of $\mathrm{OH}$ adsorbed during the measurements, the spectrum in p5 was measured first and thus has less adsorbates. To highlight the changes between the points difference spectra are displayed in Figure 6(b). The difference spectra are calculated as $\mathrm{p} i$ p1. The difference spectra for the spectra displayed in panel (a), p5, is displayed on top in panel (b). Here the increased intensity on the high binding energy side can clearly be seen, peaked at $531.1 \mathrm{eV}$. This peak is visible in all difference spectra with successively decreasing intensity as the measurement point moves closer to the (0001)-side. In Figure 5(a) the peak area integrated between $529.2 \mathrm{eV}$ and $532.8 \mathrm{eV}$, as a function of the miscut angle in the measurement point is displayed (grey crosses, interconnecting line to guide the eye). The area of this component is seen to depend almost linearly on the miscut angle, the deviation of the linear dependence in p2 is most likely due to a slight offset in the measured position. As can be seen in Figure 5(a) the area of the component scales with the density of steps as measured by STM and it is a fingerprint of under coordinated surface sites. 


\section{CONCLUSIONS}

To conclude, our STM and LEED observations show that the curved ZnO surface remains well ordered for all miscut angles. The initial (0001) terraces break up into facets of (0001) and (1014) between miscut angles of approximately 5 and $15^{\circ}$. No other facets are observed. At miscut angles above $15^{\circ}$ the surface is dominated by (1014)-facets with only a small fraction of narrow (0001)-terraces. The separation into (0001) and charge neutral (1014) nano-facets at miscut angles as low as $5^{\circ}$ is due to the non-zero dipole moment of the (0001)facets. XPS measurements reveal that the under-coordinated O-sites of the steps give rise to a characteristic component in the $\mathrm{O}$ 1s region that scales with step density. The high order of the surface and the smooth variation of the step concentration with the miscut angle makes this type of crystal highly interesting for use in future studies concerning the influence of defined step density and geometry on the catalytic properties of $\mathrm{ZnO}$.

\section{ACKNOWLEDGMENTS}

The staff at the PEARL beamline at SLS is gratefully acknowledged for their support during the XPS beamtime.

\section{REFERENCES}

${ }^{1}$ A. Mugarza and J. E. Ortega, Journal of Physics: Condensed Matter 15, S3281 (2003).

${ }^{2}$ L. S. Mende and J. L. M. Driscoll, Materials Today 10, 40 (2007).

${ }^{3}$ J. T. Yates, Journal of Vacuum Science \& Technology A: Vacuum, Surfaces, and Films 13, 1359 (1995).

${ }^{4}$ F. Tao, S. Dag, L.-W. Wang, Z. Liu, D. R. Butcher, H. Bluhm, M. Salmeron, and G. A. Somorjai, Science 327, 5967 (2010).

${ }^{5}$ X. Liu, J. Zhang, S. Wu, D. Yang, P. Liu, H. Zhang, S. Wang, X. Yao, G. Zhu, and H. Zhao, RSC Advances 2, 6178 (2012).

${ }^{6}$ E. A. Carbonio, M. J. Prieto, A. de Siervo, and R. Landers, Journal of Physical Chemistry C 118, 28679 (2014).

${ }^{7}$ M. Shipilin, J. Gustafson, C. Zhang, L. R. Merte, and E. Lundgren, Physical Chemistry Chemical Physics 18, 20312 (2016). 
${ }^{8}$ A. M. Gomez-Marin and J. M. Feli, Surface Science 646, 269 (2016).

${ }^{9}$ V. Repain, J. Berroir, S. Rousset, and J. Lecoeur, Surface Science Letters 447, L152 (2000).

${ }^{10}$ J.-S. Lee, M.-I. Kang, S. Kim, M.-S. Lee, and Y.-K. Lee, Journal of Crystal Growth 249, 201 (2003).

${ }^{11}$ K. Kuhnke and K. Kern, Journal of Physics: Condensed Matter 15, S3311 (2003).

${ }^{12}$ E. Thune, A. Boulle, D. Babonneau, F. Pailloux, W. Hamd, and R. Guinebretiere, Applied Surface Science 256, 924 (2009).

${ }^{13}$ J. E. Ortega, G. Vasseur, I. Piquero-Zulaica, S. Matencio, M. A. Valbuena, J. E. Rault, F. Schiller, M. Corso, A. Mugarza, and J. Lobo-Checa, New Journal of Physics 20, 073010 (2018).

${ }^{14}$ M. Ilyn, A. Magaña, A. L. Walter, J. Lobo-Checa, D. G. de Oteyza, F. Schiller, and J. E. Ortega, The Journal of Physical Chemistry C 121, 3880 (2017).

${ }^{15}$ A. L. Walter, F. Schiller, M. Corso, L. R. Merte, F. Bertram, J. Lobo-Checa, M. Shipilin, J. Gustafson, E. Lundgren, A. X. Brion-Rios, P. Cabrera-Sanfelix, D. Sanchez-Portal, and J. E. Ortega, Nature Communications 6, 8903 (2015).

${ }^{16}$ A. de Alwis, B. Holsclaw, V. Pushkarev, A. Reinicker, T. Lawton, M. Blecher, E. Sykes, and A. Gellman, Surface Science 608, 80 (2013).

${ }^{17}$ J. E. Ortega, M. Corso, Z. M. A. el Fattah, E. A. Goiri, and F. Schiller, Physical Review B 83, 085411 (2011).

${ }^{18}$ M. Corso, F. Schiller, L. Fernandez, J. Corcon, and J. E. Ortega, Journal of Physics: Condensed Matter 21, 353001 (2009).

${ }^{19}$ U. Diebold, S.-C. Li, and M. Schmid, Annual Review of Physical Chemistry 61, 129 (2010).

${ }^{20}$ L. A. Miccio, M. Setvin, M. Mueller, M. Abadia, I. Piquero, J. Lobo-Checa, F. Schiller, C. Rogero, M. Schmid, D. Sanchez-Portal, U. Diebold, and J. E. Ortega, Nano Letters 16, 2017 (2016).

${ }^{21}$ C. Noguera, Journal of Physics: Condensed Matter 12, R367 (2000).

${ }^{22}$ H. Zheng, M. Gruyters, E. Pehlke, and R. Berndt, Physical Review Letters 111, 086101 (2013).

${ }^{23}$ K. S. Chan, L. Vines, L. Li, C. Jagadish, B. G. Svensson, and J. Wong-Leung, Applied Physics Letters 106, 212102 (2015). 
${ }^{24}$ E. Grånäs, M. Busch, B. Arndt, M. Creutzburg, G. D. L. Semione, A. Schaefer, J. Gustafson, V. Vonk, H. Grönbeck, and A. Stierle, "Role of hydroxilation for the atomic structure of a non-polar vicinal zinc oxide," (2019), in preparation.

${ }^{25}$ A. Stierle, T. F. Keller, H. Noei, V. Vonk, and R. Roehlsberger, Journal of large-scale research facilities $\mathbf{2}$, A76 (2016).

${ }^{26}$ D. Necas and P. Klapetek, Central European Journal of Physics 10, 181 (2012).

${ }^{27}$ M. Muntwiler, J. Zhang, R. Stania, F. Matsui, P. Oberta, U. Flechsig, L. Patthey, C. Quitmann, T. Glatzel, R. Widmer, E. Meyer, T. A. Jung, P. Aebi, R. Fasel, and T. Greber, Journal of Synchrotron Radiation 24, 354 (2017).

${ }^{28}$ M. H. von Hoegen, Zeitschrift für Kristallographie - Crystalline Materials 214 (1999).

${ }^{29}$ I. K. Robinson, E. Vlieg, and K. Kern, Physical Review Letters 63, 2578 (1989).

${ }^{30}$ O. Dulub, L. A. Boatner, and U. Diebold, Surface Science 519, 201 (2002).

${ }^{31}$ S. Rousset, V. Repain, G. Baudot, Y. Garreau, and J. Lecoeur, Journal of Physics: Condensed Matter 15, S3363 (2003).

${ }^{32}$ R. Magri, S. K. Gupta, and M. Rosini, Physical Review B 90 (2014).

${ }^{33}$ J. Wollschläger, Surface Science 383, 103 (1997).

${ }^{34}$ H.-C. Jeong and E. D. Williams, Surface Science Reports 34, 171 (1999). 


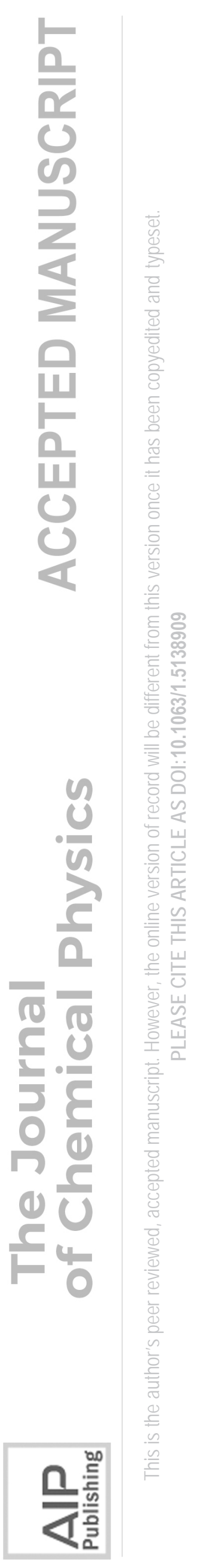

$\begin{array}{lll}\text { (b) } \mathrm{ZnO}(0001) \quad \odot \mathrm{O} & \mathrm{Zn} & \text { (c) } \mathrm{ZnO}(10 \overline{1} 4)\end{array}$
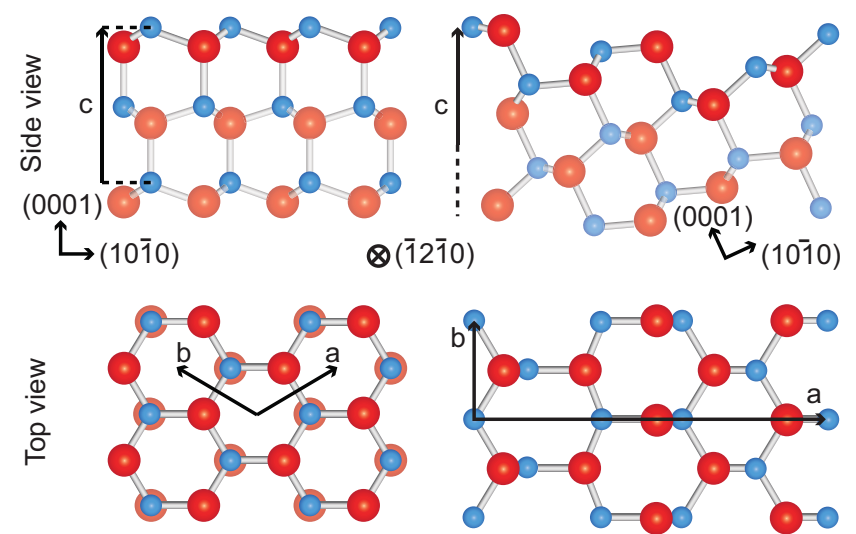

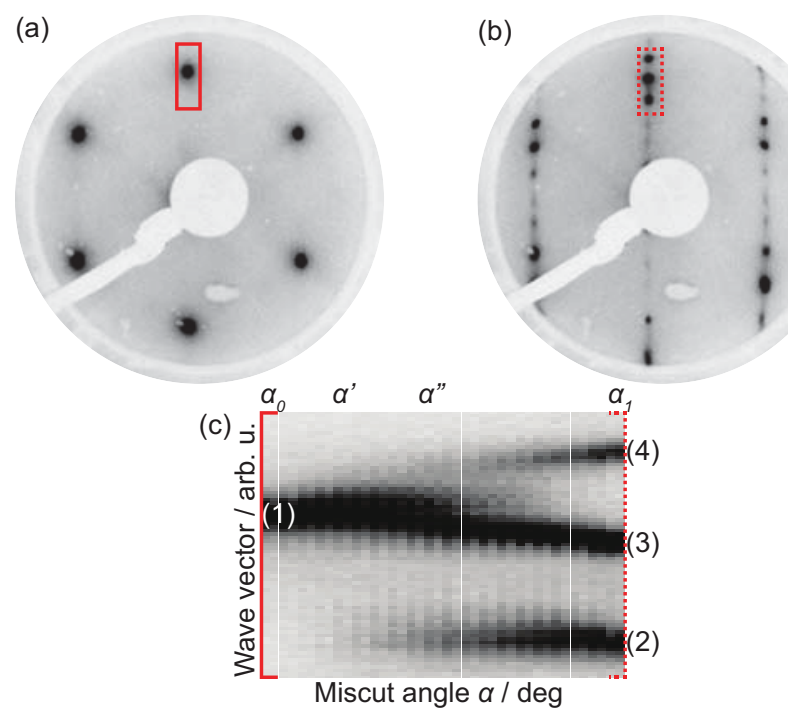

(d) $\mathrm{ZnO}(0001)$

(e) $\mathrm{ZnO}(10 \overline{1} 4)$

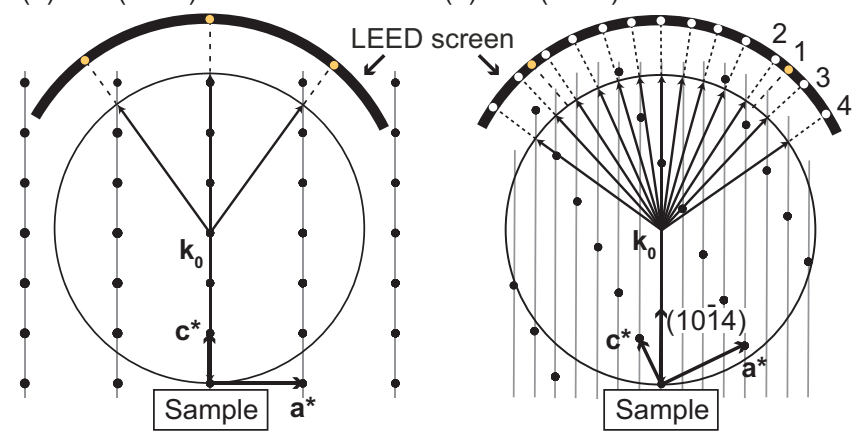

은 
(a)

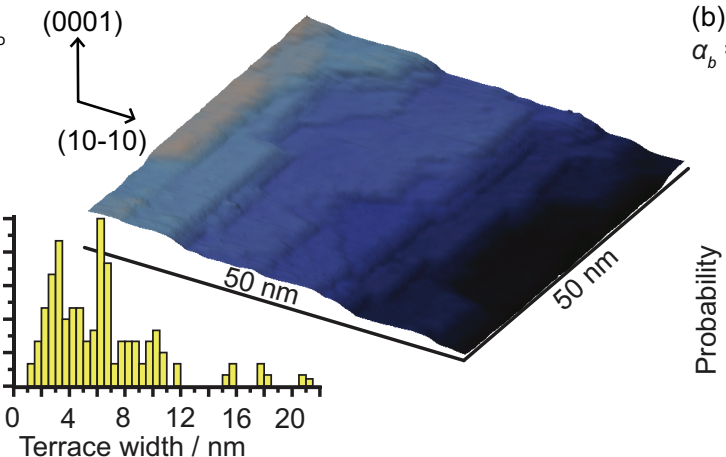

(b)

$$
a_{b}=9^{\circ}
$$

(c)

$\alpha_{c}=11^{\circ}$ (d)

$\alpha_{d}=15^{\circ}$

$\frac{3}{2}$
$\frac{2}{0}$
$\frac{0}{0}$
$\frac{0}{0}$
0

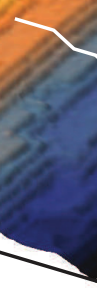
Terrace width / nm
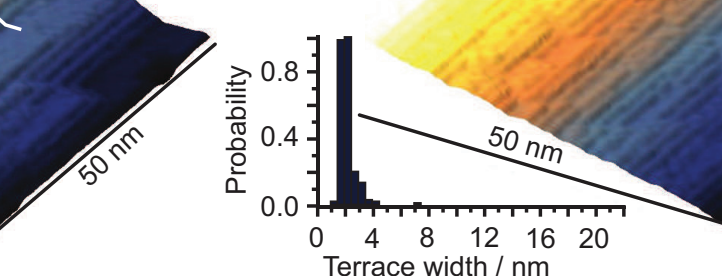

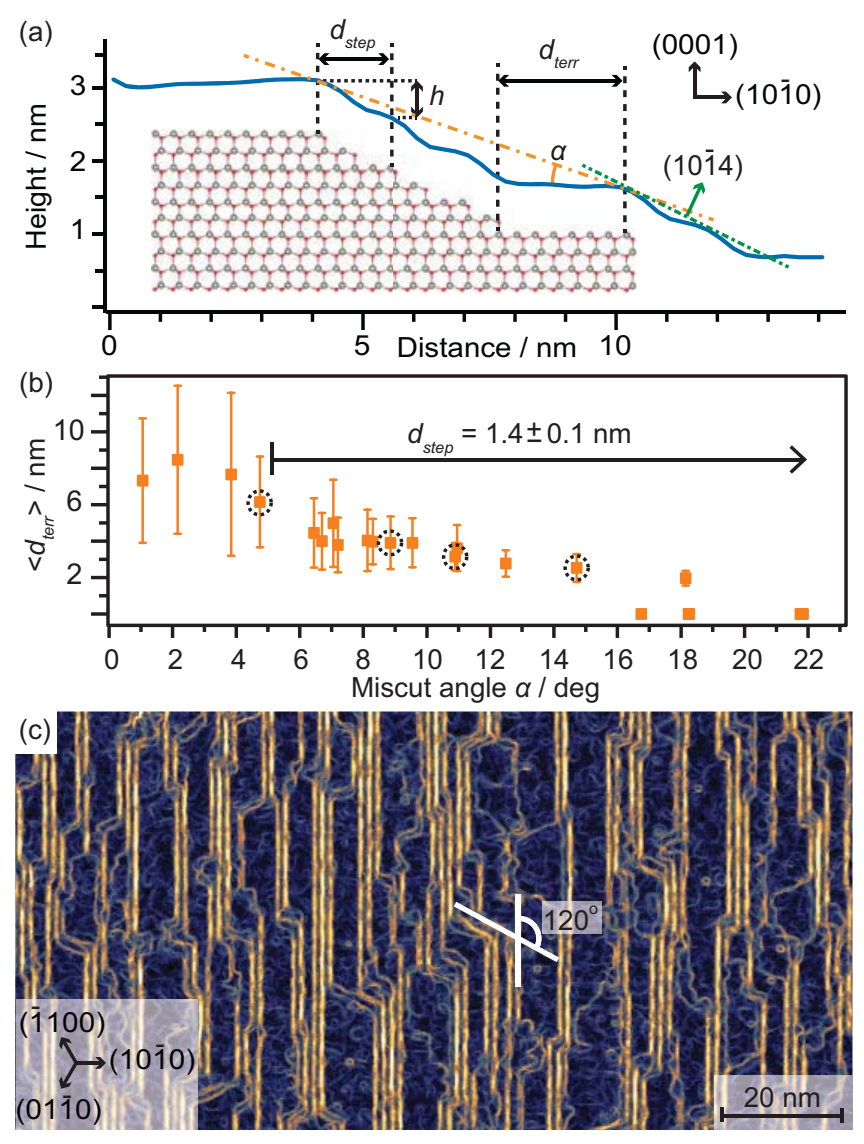

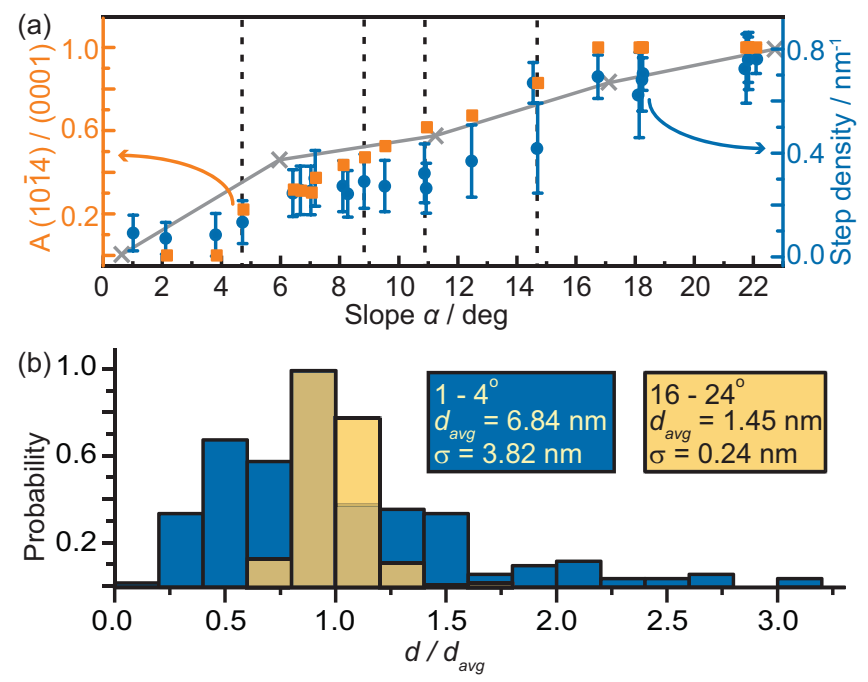
(a) $\mathrm{O} 1 \mathrm{~s} \quad \mathrm{hu}=650 \mathrm{eV}$

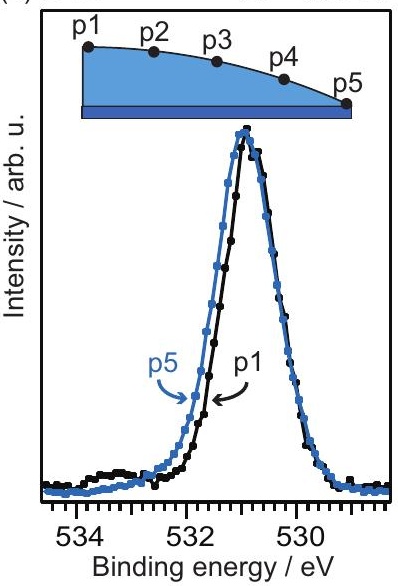

(b) 01 s difference spectra

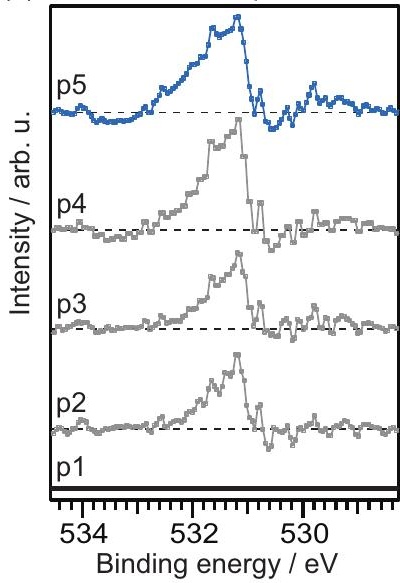

